\title{
Royauté et reconnaissance du chef par le serpent
}

\author{
Marie-José Tubiana
}

\section{OpenEdition}

Journals

Édition électronique

URL : http://journals.openedition.org/span/965

DOI : $10.4000 /$ span.965

ISSN : 2268-1558

\section{Éditeur}

École pratique des hautes études. Sciences humaines

\section{Édition imprimée}

Date de publication : 1 décembre 1990

Pagination : 189-205

ISSN : 0294-7080

\section{Référence électronique}

Marie-José Tubiana, «Royauté et reconnaissance du chef par le serpent », Systèmes de pensée en Afrique noire [En ligne], 10 | 1990, mis en ligne le 02 juillet 2013, consulté le 19 avril 2019. URL : http:// journals.openedition.org/span/965; DOI : 10.4000/span.965

Ce document a été généré automatiquement le 19 avril 2019

(c) École pratique des hautes études 


\title{
Royauté et reconnaissance du chef par le serpent
}

\author{
Marie-José Tubiana
}

Qu'il glisse sous vos pas, figure dans un bijou ou le décor d'une habitation, qu'il apparaisse comme protagoniste dans un conte, le serpent, en Afrique, est un des personnages de la vie quotidienne. Il siège également dans le panthéon africain et sa figuration sur un trône ou sur un sceptre ainsi que son rôle dans les rituels d'intronisation des chefs serait à examiner dans maintes sociétés. En effet, les exemples sont très nombreux où la divinité, lorsqu'elle entend se manifester, prend la forme d'un serpent, mais les faits, souvent décrits fragmentairement et hors contexte, ne sont pas toujours utilisables pour la comparaison. J'insisterai donc dans cette note sur les enquêtes que j'ai menées personnellement sur le sultanat du Wadday et sur des royaumes voisins appartenant à une aire culturelle qui apparaît une, et je donnerai par ailleurs quelques informations en forme de repérages pouvant étayer une recherche comparative.

2 Le sultanat du Wadday est un des grands royaumes de la zone sahélienne qui, de l'est à l'ouest, ont eu nom : Sennar, Dar For, Wadday, Baguirmi, Kanem, Borno, etc. L'emploi des termes arabes «sultanat » et «sultan » atteste leur islamisation. Pour le Wadday, celle-ci remonte au début du XVIIe siècle. Elle a commencé avec l'arrivée du fondateur de la dynastie actuelle, Abd el-Karim ibnou Djamé, dont le successeur, vingt-deuxième de la lignée, est le sultan Ibrahim Urada, intronisé en 1977 à Abbéché comme chef du « sultanat abbasside du Wadday ». Cette islamisation a, bien entendu, modifié un certain nombre de pratiques. Mais, toute ancienne qu'elle soit, elle n'a pas détruit totalement les institutions préexistantes. Il y a eu plutôt des phénomènes de superposition ou de syncrétisme que l'enquête d'ethnologie historique permet généralement de mettre en lumière.

3 Je me contenterai, ici, d'apporter quelques éléments d'information sur l'intronisation du roi : moment où l'on assiste à la transformation définitive d'une personne ordinaire en un personnage sacré.

4 Dans le sultanat du Wadday, tout membre mâle du clan royal dont la mère appartient à un clan noble peut devenir roi. D'abord réservée à quatre clans maba, cette noblesse a englobé avec le temps d'autres clans autochtones, augmentant ainsi le nombre des 
prétendants possibles : fils, frères ou cousins paternels. Nombre encore accru lorsqu'on prend en compte les alliances multiples des souverains. Il faut donc que le candidat au trône rassemble ses partisans et élimine les autres compétiteurs. Cette opération est toujours brutale : empoisonnement ou, le plus souvent, aveuglement au fer rouge car un homme qui n'a pas son intégrité corporelle ne peut être chef. Il y a donc, après la mort d'un souverain, une période - parfois très longue car on essaye de cacher le décès le plus longtemps possible - où se révèlent tous les ressorts du politique dans une atmosphère d'intrigues, de marchandages, de violence, de combats, d'assassinats et aussi d'exhibition de puissance comme peuvent l'être les courses de chevaux, les somptueux harnachements, les vêtements des cavaliers et l'étalage de cadeaux.

5 Lorsqu'un successeur possible a triomphé de ses rivaux, il convient de le faire confirmer par la divinité et en tout premier heu par le personnage-relais qu'est l'ancêtre. Abandonnant le monde profane du politique, le chef va pénétrer alors dans le monde du sacré. La compétition s'est déroulée dans la cité, sur le fasher qui est une vaste « esplanade devant le palais ", ou autour du fasher. Ce fut à Wara jusqu'en 1850, à Abbéché ensuite. Le sacre se déroule sur une montagne.

6 L'agglomération de Wara, ancienne capitale du sultanat, est située dans une dépression entourée de montagnes. L'une d'elles, la montagne toreya, est la montagne sacrée sur laquelle doit se rendre le chef pour se faire reconnaître par l'ancêtre. La montagne se présente comme un chaos de rochers, son accès est difficile, lent. Le chef grimpe au sommet de la montagne, seul, et s'y retire pendant sept jours; en ce lieu, en ce temps, dans une abstinence totale - sexuelle et alimentaire - il entre en contact avec le monde du sacré qui se manifeste sous la forme d'un serpent. Ce serpent est l'ancêtre du clan royal ou plutôt la manifestation de cet ancêtre avec lequel il est indispensable que le roi ait un contact physique. Si le serpent se manifeste, la reconnaissance a lieu. Si le serpent ne se manifeste pas ou s'il montre son hostilité, un autre sultan devra être choisi.

7 La relation fournie par Trenga (1947: 187-190), en bura-mabang avec traduction française, nous relate l'intronisation du sultan Dudmurra qui, en 1901, fut le dernier sultan à gravir les pentes de la montagne Toreya. Nous y percevons particulièrement bien les progrès de l'islamisation avec, d'une part, la présence sur la montagne sacrée de hauts personnages religieux musulmans : imam, muezzin, faki (peut-être uniquement au pied de la montagne ?) et, d'autre part, l'ignorance manifeste de ce jeune enfant, le futur sultan, vis-à-vis des croyances de ses ancêtres. Sans aucun doute, il a été élevé selon la loi musulmane. Mais nous sentons aussi la nécessité vitale, pour les Anciens, de cette reconnaissance par l'ancêtre serpent. Ce texte se situe à un moment charnière où une religion prend le pas sur une autre. Aucun sultan ne se rendit par la suite sur la montagne sacrée. Voici le texte :

«On fit monter Doud-Mourra à Toréía et on l'installa dans une case. L'Iman et le Muezzin, eux aussi, furent installés dans leurs cases, de même que les vieillards et les notables ${ }^{1}$.

Ils restèrent longtemps à attendre; leur ancêtre ne vint pas. Cela ne leur plut pas : «Pourquoi ne vient-il pas? Refuse-t-il le Sultan?»

Trois jours se passèrent; il ne vint pas; cela leur déplut beaucoup. Il vint le quatrième jour; le Sultan dormait à l'heure de la sieste. Les maitres des Coutumes se réjouirent grandement et les notables de dire : "Comment donc? S'il monte sur lui cet enfant aura peur, et s'il a peur il le tuera. Approchons-nous ».

Ils s'approchèrent; le Sultan dormait; l'ancêtre vint et monta sur ses jambes; le Sultan qui dormait ne l'entendit pas; il descendit alors, se coucha sur le tapis et plaça sa queue sur le Sultan. Celui-ci l'entendit, leva la tête et aperçut un serpent ; il 
prit doucement son sabre et tout en le regardant il dégaina son arme pour l'en frapper. Les gens qui se trouvaient dehors dirent : « Ne frappe pas! ne frappe pas!» Le Sultan alors laissa son arme ; leur ancêtre se mit violemment en colère ; ses yeux sortirent démesurément ; il darda sa langue ; il devint énorme.

Les maîtres des Coutumes lui dirent : « Ne lui fais pas (de mal); c'est votre enfant ». Il resta, alors, tranquille.

Les maîtres des Coutumes parlèrent, parlèrent longuement et l'ancêtre s'en retourna. Doud-Mourra envoya alors dire aux lettrés (faki) de faire des prières; ce qu'ils firent. Sept jours se passèrent et l'ancêtre ne revint pas; ils mirent fin aux cérémonies, descendirent de la montagne et distribuèrent de nombreuses aumônes (sadaka).

Doud-Mourra partit pour Abbéché où il tomba malade de la peur qu'il avait eue à Ouara ».

Cette relation entre le chef et l'ancêtre du clan se manifestant sous la forme d'un serpent, nous l'avons retrouvée dans bien d'autres petits royaumes ou chefferies de l'est du Tchad et du nord du Soudan. Parmi les différents rituels sur lesquels nous avons recueilli des informations deux méritent une attention particulière pour notre propos parce qu'ils éclairent le binôme roi-serpent avec plus de précision.

Lors de l'intronisation du sultan des Zaghawa kabka, au nord-est du royaume du Wadday, les événements se déroulaient en trois phases :

- 1. choix du chef par les anciens à l'intérieur du clan royal, en fonction de ses capacités mais aussi de sa richesse, révélatrice de son sens politique. Cela n'excluait pas pour autant la compétition mais elle était, semble-t-il, moins violente que pour le sultanat du Wadday, l'enjeu étant d'ailleurs moindre ;

- 2. ratification du choix par le sultan du Wadday sauf lorsque le chef se sentait assez fort pour se dispenser de cette démarche et se contentait alors d'avertir son "supérieur» de sa nomination, les deux attitudes indiquant toutefois un réel lien de vassalité;

- 3. pèlerinage du chef sur la montagne sacrée propre à son clan, le clan royal, pour y faire un sacrifice d'un type particulier. Il s'agissait d'immoler une chamelle pleine. Le foetus extrait du ventre de la bête immolée était jeté dans un trou situé au sommet de la montagne ; il était destiné à la fois à la montagne dans les entrailles de laquelle il était poussé à l'aide d'un bâton et à la manifestation visible de l'ancêtre: le serpent qui demeurait dans le trou (Tubiana, 1964 : 82-25).

10 Les informateurs ont été unanimes : si le serpent se montrait durant le sacrifice, ce serait une bonne année; si au contraire le serpent restait caché, c'était signe que l'année serait mauvaise ou que le chef mourrait; mais il n'était semble-t-il pas question de nommer un nouveau chef comme cela se passait dans le sultanat du Wadday. Lorsque le serpent était favorable, il sortait de la montagne et accompagnait les sacrifiants en glissant doucement à côté d'eux. Il venait lécher le sang du sacrifice et manger les petits morceaux de viande prélevés sur la victime et déposés en offrandes sur son passage. C'était un grand serpent blanc qui portait sur sa tête deux plumes d'autruches blanche ${ }^{2}$. Le serpent est paré de plumes d'autruche blanches, comme l'est la jeune épousée du sultan kabka, tandis que la chamelle du sacrifice porte sur la tête des plumes blanches mais aussi des noires (Tubiana, $1964: 83)^{3}$.

11 Dans une chefferie voisine du sultanat kabka, celle des Zaghawa kigé, nous avons retrouvé les différentes phases déjà signalées. Celle des compétitions politiques met l'accent encore plus ostensiblement sur l'enjeu économique: c'est le candidat capable de rassembler le plus d'animaux et d'offrir au sultan du Wadday le plus gros troupeau qui 
triomphe. Le moment de l'investiture par le serpent a fait l'objet au cours de notre enquête d'une description minutieuse qui fut mimée sur place par un des participants au dernier rituel (autour de 1910).

Le chef, choisi par un sultan étranger, se fait d'abord reconnaître par les gardiens de la montagne, sacrifie avec eux une vache dont la peau servira à recouvrir la timbale de la chefferie avant de se rendre sous un abri sous roche. Là se trouve une pierre sur laquelle il s'assoit comme sur un trône. Il attend le chef des serpents qui est noir avec une tête blanche ornée de deux plumes d'autruches blanches. S'il approuve le choix du nouveau chef, le serpent tourne autour de lui, puis se couche à ses côtés. Si au contraire, il est mécontent, il gonfle ses joues et le chef s'enfuit. Un chef qui n'est pas reconnu par le serpent n'est pas mis à mort, mais est évincé. Par contre, un chef agréé par le serpent ne pourra jamais être destitué. C'est seulement après la reconnaissance du chef par le serpent que la timbale royale, insigne du pouvoir, est recouverte d'une nouvelle peau. Dans le cas de non-reconnaissance et de nomination d'un nouveau chef, une autre vache sera sacrifiée, une nouvelle peau préparée pour recouvrir la timbale. Tout s'articule donc autour de la reconnaissance par le serpent (Tubiana, 1964 : 89-96).

Que représente le serpent dans les rituels d'intronisation que nous venons brièvement de décrire et quels sont les liens entre la montagne, le chef et le serpent? Le chef est l'élément vivant d'une longue lignée détentrice du pouvoir, capable de fournir une généalogie en partie historique, en partie mythique qui le rattache à un ancêtre fondateur. La montagne sacrée est le lieu où cet ancêtre, premier occupant et maître du sol, est venu s'établir, à moins qu'il ne soit né de cette montagne. On invoque ce haut-lieu en le nommant er-guru, "notre grand-père ", " notre ancêtre ", ou bien manda x ou y en se référant au nom même de la montagne. Le terme manda désigne l'esprit qui habite un lieu (ici montagne, ailleurs arbre) - sorte de genius loci - et plus largement tout ce qui est sacré. Le serpent qui hante la montagne, c'est la manifestation de l'ancêtre comme être immortel. Il apparaît dans les grands moments de la vie du clan et donc dans celui, majeur, de la reconnaissance du chef. On s'adresse à lui avec les mêmes termes que ceux employés pour parler à la montagne : er-guru ou manda. Ancêtre, montagne et serpent font partie d'un même ensemble identifié, localisable, chargé de sacré, capable à son tour de sacraliser un de ses descendants.

En dehors des grands rituels d'intronisation qui sont, rappelons-le, des rituels de fertilité conférant au chef le devoir et la capacité d'amener la pluie, donc la prospérité, sur son pays, les sacrifices faits annuellement par les différents chefs de clan dans le même but, soit sur la montagne, soit au pied de l'arbre du clan, sont destinés aussi à un serpent (parfois à deux). Le sang, les os ou le coeur de la victime, ainsi que des boules de farine de mil, du sel ou du beurre lui sont offerts. Il vit dans la montagne ou dans l'arbre. Il est dit " fils de la montagne ", le tuer entraîne la mort des hommes et la stérilité de la terre (Tubiana, $1964: 113,124$ ).

15 Le serpent-ancêtre a été signalé dans de nombreuses populations du Dar For : chez les Mun du djebel Mun où le serpent apporterait des présages depuis le djebel Nyéri, ancienne résidence des sultans du Tama, jusqu'au djebel Mun (le djebel Nyéri serait mâle et le djebel Mun femelle); chez les For des dar Kerné et Dima, ainsi qu'au Kordofan, chez différents groupes Nuba établis autour des montagnes (Tubiana, 1964: 188-91 et 95-96 s'appuyant sur les travaux de MacMichaël, Davies, Beaton et Seligman). En 1990, enfin, nous avons retrouvé la trace d'un gros serpent blanc sur le hadjer kudjunu chez les Dadjo du Dar Sila. Les similitudes sont frappantes d'une population à l'autre mais les différences 
sont aussi très grandes sans doute sous diverses influences dont celle prépondérante de l'Islam. En effet, si l'ancêtre se manifeste le plus souvent sous la forme d'un serpent, il peut aussi dans certains cas se manifester sous celle de javelots; la charge de faiseur de pluie peut changer de mains et passer des mains du chef à celles d'un spécialiste, mais surtout le statut même du serpent peut se modifier: il devient parfois un «mauvais génie » ou un « démon ».

La ville de Wara et la montagne toreya selon le tracé original du Cheykh Mohammed El-Tounsy

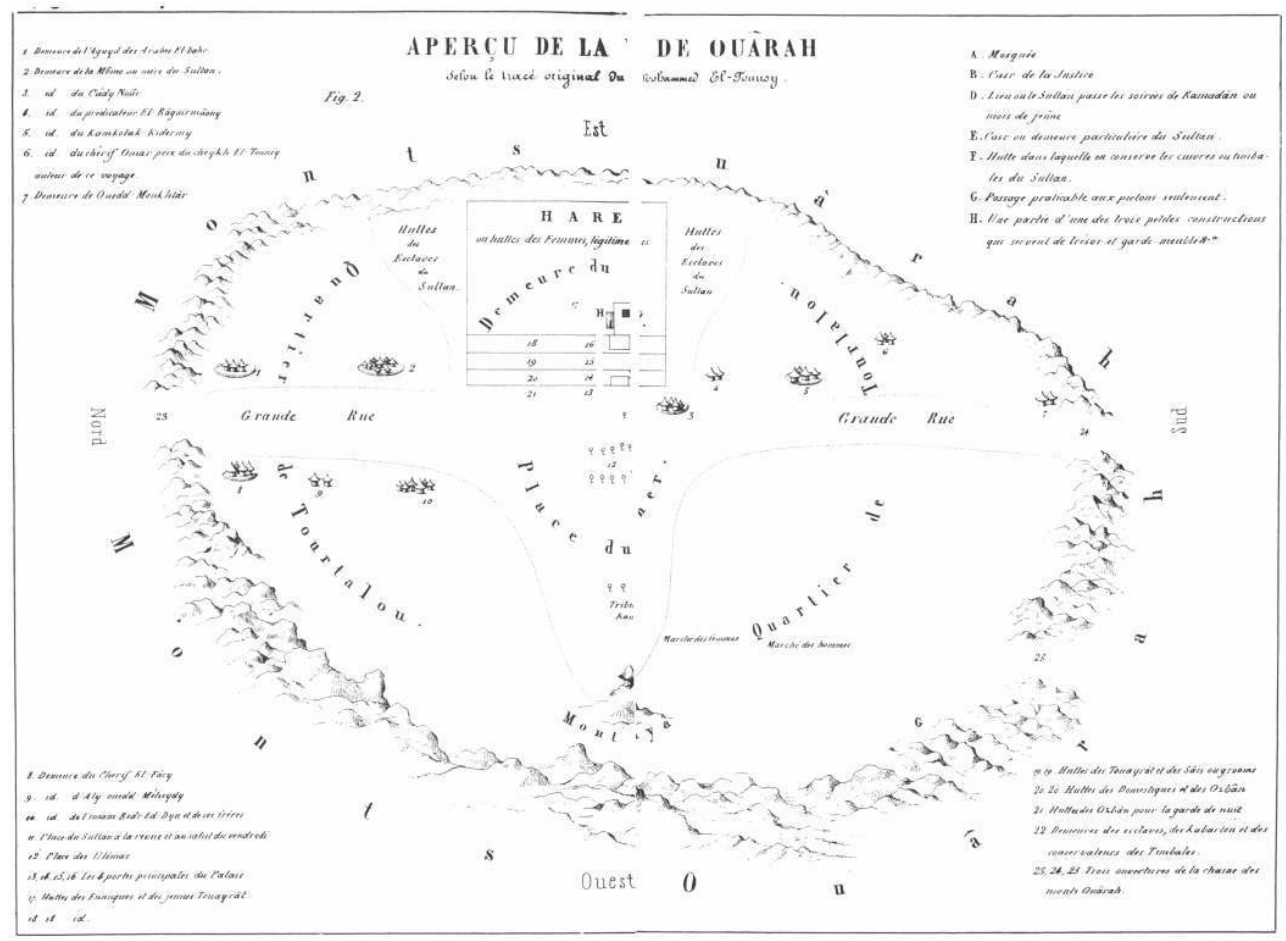

(Gravure extraite de El-Tounsy M., Voyage au Ouadây, pl. III).

Si l'on cherche ailleurs des éléments de comparaison, les margay des Hadjeray se présentent sous des traits comparables aux manda des Zaghawa. Elles se manifestent parfois aux chefs religieux sous la forme d'un python (ou d'un varan) mais il ne semble pas toutefois que celui-ci jouait un rôle dans la reconnaissance des chefs (Vincent, 1975 : 76 et $89-90$ ).

17 Chez les Bilala du Fitri, la marge de Gollo, village de pêcheurs près du lac, se manifeste sous la forme d'un gros serpent, Gashé May, métamorphose d'une jeune femme dont les actuels sultans de Yao seraient les descendants (Hagenbucher, 1968 : 57-58).

Dans les royaumes kotoko, la description des rites liés à l'intronisation du chef et la reconnaissance de celui-ci par les trois varans propriétaires du sol offrent des éléments comparables. Le varan satisfait « se frotte contre les parois du trou » où il réside, ou bien " pose sa tête sur le trône, regarde le souverain et rentre ». Dans tous les cas il doit se montrer (Griaule \& Lebeuf, 1951: 6-30). A.-M. D. Lebeuf signale que le Prince doit nécessairement être agréé par un varan et un serpent qui sortent de leur tanière et acceptent l'offrande de nourriture qui leur est faite faute de quoi on procède à une nouvelle élection. Le serpent, un python, est associé à l'eau et le varan à la brousse (1969 : 234-243). 
Cuoq (1975: 98), rapportant Al-Bakri, signale que dans l'un des royaumes vassaux de Ghana, le Jafunu, le choix d'un futur roi est soumis à une " consultation » préalable d'un serpent qui fixe également la durée de son règne.

Jean Bazin (1988: 422-423) signale que le roi tarawure de Jaa (région de Segu) avait, paraît-il, dans sa chambre secrète une jarre pleine d'eau dans laquelle était gardé un reptile. La consommation de cette eau permettait au corps royal de se régénérer périodiquement. Il rapporte également que selon les Tarawure de Bengene (village royal de la région de Segu, au Niger, où il a mené une enquête) le pouvoir du roi dérive d'un secret, dont il est le seul dépositaire, qui lui permet de prédire l'avenir en consultant le fameux serpent (id. : 434-435).

Dans les royaumes gambiens de Wuli et de Nyani, le « fétiche royal » est aussi un serpent (Cissoko, 1968 : 124).

$\mathrm{Au}$ Dahomey, le roi est associé à Dan le serpent-arc-en-ciel comme on peut le voir sur un bas-relief du palais de Ghezo, roi d'Abomey (Waterlot, 1926 : pl. IX).

23 Mentionnons aussi Lébé, l'ancêtre le plus ancien auquel se réfère la mythologie dogon. Il mourut sous une forme humaine - jusque-là les hommes étaient immortels - fut enterré et quand les Dogon quittèrent le pays et ouvrirent sa tombe pour emporter avec eux les os de leur aïeul, ils trouvèrent un grand reptile vivant qui les suivit dans leur voyage jusqu'aux falaises de Bandiagara. Depuis, le serpent mythique est censé venir chaque nuit lécher le corps du Hogon, son prêtre et descendant et, à l'aube, regagner son séjour souterrain, chez les génies, en se glissant par une faille entre deux rochers. Le Hogon ne se lave jamais (Paulme, 1988 : 25, 200).

Autres jalons, plus lointains dans le temps ou dans l'espace : dans une gravure qui dépeint le couronnement du roi de Ouidah on voit au centre du dessin la case du serpent et le serpent lui-même qui s'y rend apparemment précipitamment (Labat, 1731, II : 70). L'auteur nous décrit par ailleurs les différentes processions faites à la maison du grand Serpent qualifié de «principale divinité du païs». Les Grands et le peuple s'y rendent avant le couronnement du souverain pour demander un prince «qui soit aussi bon et équitable que son prédécesseur, qui fasse fleurir le commerce, qui soit un religieux observateur des lois » (p. 55), et une fois la cérémonie accomplie, après quinze jours de longues fêtes, a lieu une procession solennelle à la «maison» ou «temple du grand Serpent » (p. 61). Dans les deux cas il ne semble pas qu'il y ait vraiment reconnaissance au sens où nous l'entendons mais plutôt demandes d'une part et actions de grâces de l'autre. Un autre auteur (Casimir Agbo, 1959: 15) nous dit que Kyassé, le deuxième roi Houéda fondant au XVIe siècle ce qui deviendra la ville de Ouidah, consacra sa ville au " fétiche » Dagbé qui est le serpent python, lequel est devenu le « totem des Houéda ».

Dans l'art du Bénin des XVIIe et XVIIIe siècles, la représentation du serpent est omniprésente : plaques de laiton, vases, sceptres, défenses d'ivoire surplombant les têtes commémoratives des oba, etc. (Duchâteau, $1990: 63$ ).

Comment enfin ne pas être tenté de regarder vers l'Ethiopie où le mythe du roi-serpent et de sa mise à mort symbolise la destruction du paganisme et où l'histoire du serpent est conçue comme la «préhistoire » de la dynastie salomonide (Perret-Denais, 1983 : 117-150) ou encore vers Méroé (trois siècles avant Jésus-Christ - quatre siècles après) où un magnifique serpent à tête de lion et bras humains figure sur les piliers monumentaux d'un temple ou d'un palais de Wad ben Naqa. L'association serpent-lion mérite aussi de retenir notre attention dans un contexte africain où le chef est souvent symbolisé par le 
lion. Ainsi lors du rituel d'intronisation du chef kigé, auquel nous avons fait référence, c'est en se comportant comme un lion que le chef s'approche de la vache qu'il va sacrifier. Il avance à quatre pattes en rugissant et en bondissant et lorsqu'il est à proximité de la vache il la frappe sur le dos comme le ferait un lion. La grotte où il se rend pour être investi par le serpent se nomme indifféremment erbet giRi ou ina giRi, c'est-à-dire « l'abri du lion » ou «l'abri du chef », ce qui confirme la synonymie entre lion et chef (Tubiana, 1964 : 92). Sur ce point aussi des enquêtes mériteraient d'être menées en Afrique et hors d'Afrique comme nous invite à le faire la très riche étude d'Elena Cassin sur « Le Roi et le Lion» (1981) en Mésopotamie ancienne. Cette recherche conduite à travers noms propres, hymnes royaux et bas-reliefs décorant un palais fait apparaitre le roi comme un lion mais aussi comme un chasseur de lions qui, par la mise à mort de son rival dans un combat rituel, assimile la force souveraine du lion sur le monde sauvage. Dans le même article une brève mention signale que la figure du roi peut se trouver associée également au serpent (p. 356) mais seule la relation roi-lion est étudiée. Peut-on avec l'apparition du serpent suggérer l'hypothèse d'une extension du pouvoir royal au monde souterrain?

Site méroïtique de Wad ben Naqa.

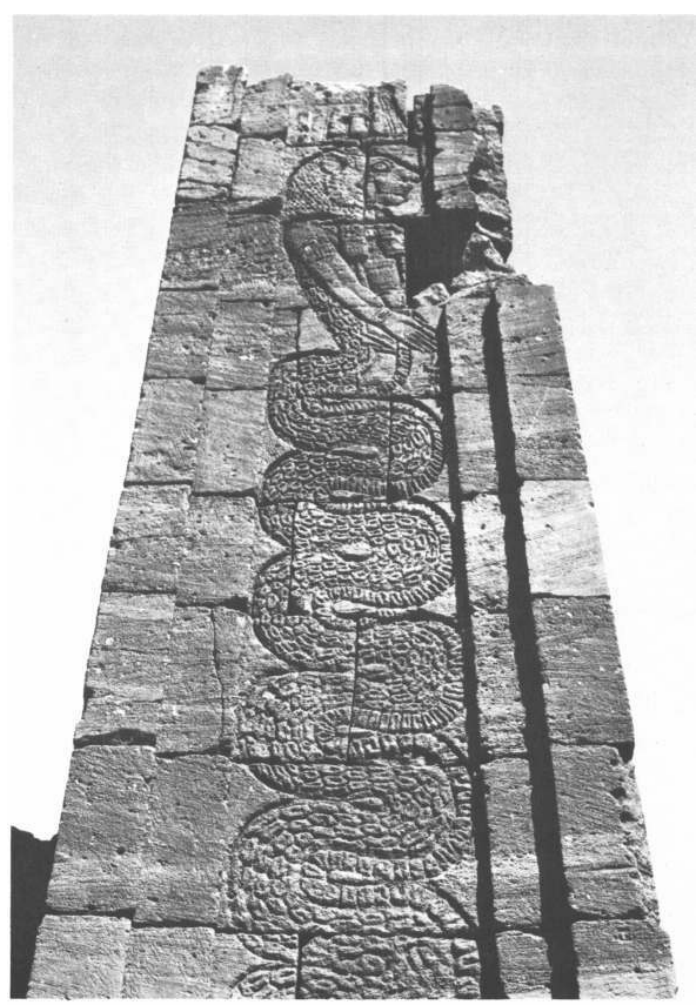

Cl. M.-J. Tubiana, novembre 1979.

27 Que représente enfin le serpent qui vient goûter les mets qu'Enée a posés en offrande sur le tombeau de son père ? Est-ce un génie du lieu? Est-ce le messager du père ? Est-ce le père? (Dumézil, 1966 : 57-58).

Ma préoccupation à travers ces quelques remarques a été d'essayer d'appréhender le passage du profane au sacré. Une séparation de deux ordres est apparue très nettement: dans le temps avec la succession des faits, et dans l'espace avec le déplacement des personnes de la plaine vers le sommet de la montagne, des lieux habités vers les lieux inhabités. La place et le rôle tenu par le serpent dans la reconnaissance du chef a fait 
apparaître le roi non pas comme une personne sacrée mais comme une personne «sacralisée » à un certain moment de son parcours initiatique et c'est à partir de ce moment-là, et de là seulement, que le chef est investi d'un pouvoir sur la nature et sur la fertilité en général.

Si l'on s'en tient maintenant aux enquêtes que j'ai pu mener personnellement, la collecte de faits comparables dans une aire culturelle commune pourrait faire penser à un schéma d'emprunt: le sultanat waddayen dominant offrant un modèle aux chefferies périphériques plus ou moins dépendantes. Mon sentiment est que les choses ne se sont pas passées ainsi et que nous avons affaire à un substrat préislamique commun. Ce fond préislamique renvoie aux prédécesseurs d'Abd al-Karim, c'est-à-dire aux Tundjur vaincus par lui, et qui au XVIe siècle dominèrent le Dar For et le Wadday. Les informations de Palmer (1928 : 24-28) et les enquêtes que j'ai menées dans les montagnes du Dar Furnung, au Soudan, encore de nos jours habitées par des Tundjur, apportent de nombreux éléments qui montrent que les anciens lieux de culte et les rituels des Tundjur vaincus ont été repris par les vainqueurs. Il m'apparaît maintenant probable que le serpentancêtre reconnaissant le chef était présent dans la religion tundjur. Et si l'on reprend le parallèle avec l'Ethiopie amorcé brièvement quelques lignes plus haut, là aussi, on peut supposer que l'histoire du serpent a pu être le grand mythe-fondateur de la monarchie aksoumite, témoin de l'importance et de la permanence du substrat agaw dans la civilisation éthiopienne.

Les rituels d'intronisation que nous avons évoqués ont disparu sous la pression de l'Islam et c'est parce que des témoins subsistent encore que nous pouvons comprendre leur fonctionnement et leur importance. Mais le respect qui entoure le serpent-ancêtre est toujours aussi vivace et peut être confronté à l'actualité la plus brûlante. Des faits qui m'ont été rapportés dernièrement, en 1987 et en 1989, montrent la permanence du culte du serpent dans le nord-est du Tchad. Plusieurs versions d'une même histoire ou plusieurs histoires relatent les malheurs qui se sont abattus sur des militaires du FROLINAT qui, venus s'installer dans des grottes ou des abris situés sur une montagne sacrée (en particulier la montagne deni ou montagne « du serpent»), ont tué le serpent malgré la mise en garde de la gardienne de cette montagne (qui remplit toujours ses fonctions en faisant au serpent des libations de lait, de beurre ou de bouillie de mil). Les châtiments varient selon les versions mais il est toujours question de la mort des hommes. Quelquefois c'est la montagne elle-même qui détache un énorme rocher écrasant les hommes, obstruant entièrement la grotte et emmurant le dépôt d'armes qui y était caché.

\section{BIBLIOGRAPHIE}

Agbo, dit Alidji, C., 1959, Histoire de Ouidah du XVIe au XXe siècle, Les Presses Universelles.

Bazin, J., 1988, « Princes désarmés, corps dangereux. Les « rois-femmes de la région de Segu », C ahiers d'Etudes africaines, 111-112, XXVIII, 3/4, 375-441.

Cassin, E., 1981, « Le Roi et le Lion », Revue de l'Histoire des Religions, CXCVIII, 4 
Cissoko, S.-M., 1968, « Prophéties de roi Mandingue : Mansa Dâli ! », Notes africaines, CXX, 123-124.

Cuoq, J.-M., 1975, Recueil des sources arabes concernant l'Afrique occidentale du Ville au XVe siècle (Bilad al-Sudan), Paris, C.N.R.S.

Denais, M. et Perret, M., 1983, « La mort du serpent ». Guirlande pour Abba Jérôme, Le Mois en Afrique, 117-152.

Du château, A., 1990, Bénin trésor royal. Collection du Museum fur Völkerkunde Vienne, Dapper, Paris.

Dumézil, G., 1966, La religion romaine archaïque, Paris, Payot.

Griaule, M. et Lebeuf, J.-P., 1951, « Fouilles dans la région du Tchad », Journal de la Société des Africanistes, XXI, 1, 1-96.

Hagenbucher, F., 1968, « Notes sur les Bilala du Fitri », Cahiers ORSTOM (Série Sciences Humaines), $\mathrm{V}, 4,39-69$.

Labat (Père), J.-B., 1731, Le voyage du Chevalier Des Marchais en Guinée, isles voisines et à Cayenne, fait en 1725, 1726 et 1727, Amsterdam, tome II, 292 p.

Lebeuf, A.-M.D, .1969, Les principautés kotoko. Essai sur le caractère sacré de l'autorité, Paris, CNRS.

Paulme, D., 1940, Organisation sociale des Dogon, Paris, Domat-Montchrestien (réédité en 1988).

Palmer, H.R., 1928, Sudanese Memoirs, 3 vol., Lagos, Government Printer.

El-Tounsy, Mohammed ibn Omar, 1851, Voyage au Ouadây. Traduit de l'arabe par le Dr Perron, Paris, Duprat.

Trenga, G., 1947, Le Bura-Mabang du Ouadaï, Paris, (Travaux et Mémoires de l'Institut d'Ethnologie, XLIX).

Tubiana, M.-J., 1964, Survivances préislamiques en pays zaghawa, Paris, (Travaux et Mémoires de l'Institut d'Ethnologie LXVII).

Vincent, J.-F., 1975, Le pouvoir et le sacré chez les Hadjeray du Tchad, Paris, Anthropos.

Waterlot, E.-G., 1926, Les Bas-Reliefs des Bâtiments royaux d'Abomey (Dahomey), Paris, (Travaux et Mémoires de l'Institut d'Ethnologie I).

\section{NOTES}

1. Les vieilles gens qui accompagnent Doud-Mourra sont les vieilles femmes et les vieillards gardiens de la montagne.

2. Les éventails en plumes d'autruches, noires, figurent parmi les insignes royaux associés aux timbales. Des serviteurs les font tournoyer au-dessus des timbales ou devant le sultan lors des danses accompagnant les grandes cérémonies (observées chez les Zaghawa et en mars 1990 encore chez les Dadjo de Goz Beida).

3. Plumes noires et blanches proviennent du plumage des autruches mâles. Il se peut que les plumes blanches de la queue et du bout des ailes, plus rares et plus longues, soient plus valorisées. Le plumage de la femelle est gris-brun. 
INDEX

Mots-clés : royauté sacrée, serpent, varan, lion, fétiche

Index géographique : Sultanat de Wadday, Tchad, Soudan

\section{AUTEUR}

MARIE-JOSÉ TUBIANA

UA 1024 CNRS-INALCO 